\title{
Correction: Krauss, J., et al. Epichloë Endophyte Infection Rates and Alkaloid Content in Commercially Available Grass Seed Mixtures in Europe. Microorganisms 2020, 8, 498
}

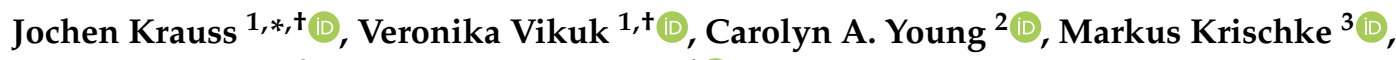 \\ Martin J. Mueller ${ }^{3}$ and Katja Baerenfaller 4 (D) \\ 1 Department of Animal Ecology and Tropical Biology, University of Würzburg, 97074 Würzburg, Germany; \\ veronika.vikuk@uni-wuerzburg.de \\ 2 Noble Research Institute LLC, Ardmore, OK 73401, USA; cayoung@noble.org \\ 3 Department of Pharmaceutical Biology, Metabolomics Core Unit, University of Würzburg, \\ 97082 Würzburg, Germany; krischke@biozentrum.uni-wuerzburg.de (M.K.); \\ martin.mueller@biozentrum.uni-wuerzburg.de (M.J.M.) \\ 4 Swiss Institute of Allergy and Asthma Research (SIAF), University of Zurich, and Swiss Institute of \\ Bioinformatics (SIB), 7265 Davos, Switzerland; katja.baerenfaller@siaf.uzh.ch \\ * Correspondence: j.krauss@uni-wuerzburg.de; Tel.: +49-(0)931-318-2382 \\ + These authors contributed equally to this work.
}

Received: 13 October 2020; Accepted: 19 October 2020; Published: 21 October 2020

The authors wish to make the following correction to this paper [1]:

After the publication of the manuscript, the authors recognized that there is a discrepancy in Table 1 in five seed mixture compositions (S_10,S_13,S_17, S_29 and S_33), due to differences between the information in catalogs and the actual product labels. The authors changed the seed compositions and suppliers, where necessary, to match with product labels on the seed mixture packages. We also deleted two sentences from the discussion regarding speculations on possible infections of the seed variety NEW ORLEANS, which was not a component in any of the tested seed mixtures.

We changed Table 1 and present the correct varieties here.

Table 1. Composition of grassland seed mixtures, bold indicate seed mixtures with infections of Epichloë spp. and detected vertebrate toxic alkaloids. Letters in brackets after the product name indicate if seed mixtures are mainly used as forage grass (F) or turf grass (T) according to supplier information. Supplier names are in italic. Changed information is highlighted in grey.

\begin{tabular}{|c|c|c|c|c|}
\hline ID & Perennial Ryegrass Varieties & Tall Fescue Varieties & Other Grasses & Product \\
\hline S_10* & $\begin{array}{c}30 \% \text { BARCLAY II } \\
\text { 15\% BAREURO } \\
20 \% \text { BARLICUM } \\
20 \% \text { BARLIBRO } \\
15 \% \text { BAROMARIO }\end{array}$ & - & - & $\begin{array}{l}\text { Regenerations-Mischung RPR, } \\
\text { Eurogreen, Barenbrug (T) }\end{array}$ \\
\hline S_11 & $\begin{array}{l}\text { Unknown variety in unknown } \\
\text { percentage }\end{array}$ & - & $\begin{array}{c}\text { Poa pratensis, Festuca pratensis, } \\
\text { Dactylis glomerata, Phleum pratense, } \\
\text { Festuca rubra, Agrostis }\end{array}$ & $\begin{array}{l}\text { Gräsermischung Weidesaat, } \\
\text { Kräuterwiese }(\mathrm{F})\end{array}$ \\
\hline S_12 & $\begin{array}{c}25 \% \text { lawn type, unknown variety } \\
25 \% \text { pasture type, unknown } \\
\text { variety }\end{array}$ & - & $\begin{array}{l}20 \% \text { Poa pratensis, } \\
20 \% \text { Phleum pratense, } \\
10 \% \text { Festuca rubra }\end{array}$ & Country Horse 2117, DSV (F) \\
\hline
\end{tabular}


Table 1. Cont.

\begin{tabular}{|c|c|c|c|c|}
\hline ID & Perennial Ryegrass Varieties & Tall Fescue Varieties & Other Grasses & Product \\
\hline S_13* & $\begin{array}{c}14 \% \text { CARNAC } \\
21 \% \text { EURODIAMOND } \\
7 \% \text { DOUBLE } 4 \mathrm{n} \\
8 \% \text { FABIAN } 4 \mathrm{n} \\
8 \% \text { CSI CORSICA } \\
17 \% \text { ZÜRICH }\end{array}$ & - & $25 \%$ Poa pratensis & Regeneration Highspeed, UFA (T) \\
\hline S_14 & - & $100 \%$ LIPALMA & - & Camena Samen (F) \\
\hline S_15 & $\begin{array}{c}33 \% \text { MATHILDE } \\
34 \% \text { WADI } \\
33 \% \text { BELIDA }\end{array}$ & - & - & $\begin{array}{l}\text { Elite Gvo (ELITE Grünland Nr. 5), } \\
\text { Rudloff (F) }\end{array}$ \\
\hline S_16 & $\begin{array}{l}20 \% \text { EURODIAMOND, } \\
15 \% \text { SIRTAKY }\end{array}$ & $\begin{array}{l}15 \% \text { BARCESAR, } \\
35 \% \text { MEANDRE }\end{array}$ & $15 \%$ Poa pratensis & Reitbahn, UFA (T) \\
\hline S_17* & $25 \%$ ASTONHOCKEY & $20 \%$ HYKOR & $\begin{array}{l}25 \% \text { Festuca pratensis, } \\
20 \% \text { Phleum pratense, } \\
10 \% \text { Poa pratensis }\end{array}$ & Country Öko 2217, DSV (F) \\
\hline S_18 & $\begin{array}{c}15 \% \text { BOYNE } \\
20 \% \text { TODDINGTON } \\
20 \% \text { INDICUS } 1 \\
15 \% \text { POLIM } \\
15 \% \text { ARUSI } \\
15 \% \text { GARBOR }\end{array}$ & - & - & Profi Nachsaat Gvo, Tystofte Fonden (F) \\
\hline S_19 & $100 \%$ KARATOS & - & - & Camena Samen (F) \\
\hline S_20 & - & - & $\begin{array}{c}7 \% \text { Agrostis capillaris, } \\
3 \% \text { Alopecurus pratensis, } \\
12 \% \text { Arrhenatherum elatius, } \\
10 \% \text { Cynosurus cristatus, } \\
10 \% \text { Dactylis glomerata, } \\
15 \% \text { Festuca rubra, } \\
1 \% \text { Holcus lanatus, } 1 \\
3 \% \text { Phleum pratense, } \\
18 \% \text { Poa pratensis, } \\
1 \% \text { Trisetum flavescens }\end{array}$ & $\begin{array}{l}\text { Heuwiese für Pferde, Appels Wilde } \\
\text { Samen }(\mathrm{F})\end{array}$ \\
\hline S_21 & $\begin{array}{c}10 \% \text { KARATOS } \\
20 \% \text { KUBUS, } \\
15 \% \text { TWYMAX }\end{array}$ & - & $\begin{array}{c}25 \% \text { Phleum pratense } \\
12 \% \text { Poa pratensis } \\
15 \% \text { Festuca rubra }\end{array}$ & Pferdeweide 1, Camena Samen (F) \\
\hline S_22 & 8\% PREMIUM & - & $\begin{array}{l}18 \% \text { Festulolium, } 18 \% \text { Phleum } \\
\text { pratense, } 15 \% \text { Festuca pratensis }\end{array}$ & Rotkleegras 91, Camena Samen (F) \\
\hline S_23 & $100 \%$ POLIM & - & - & Camena Samen (F) \\
\hline S_24 & $\begin{array}{c}12 \% \text { BELLEVUE } \\
20 \% \text { BOYNE } \\
40 \% \text { STEFANI } \\
\end{array}$ & - & $\begin{array}{l}18 \% \text { Phleum pratense } \\
10 \% \text { Poa pratensis }\end{array}$ & Pferdeweide Nachsaat, Raiffeisen (F) \\
\hline S_25 & $\begin{array}{c}20 \% \text { IVANA } \\
10 \% \text { TIVOLI } \\
20 \% \text { SW BIRGER }\end{array}$ & $20 \%$ SWAJ & $\begin{array}{c}10 \% \text { Poa pratensis } \\
20 \% \text { Phleum pratense }\end{array}$ & Pferdegreen Öko PR940, BSV Saaten (F) \\
\hline S_26 & $100 \%$ TWYMAX & - & - & Camena Samen (F) \\
\hline S_27 & $\begin{array}{l}28 \% \text { MATHILDE, } 23 \% \text { ALFAN, } \\
13 \% \text { BELIDA }\end{array}$ & - & $\begin{array}{l}10 \% \text { Festuca pratensis, } 5 \% \text { Poa } \\
\text { pratensis, } 21 \% \text { Phleum pratense }\end{array}$ & Elite 20, Rudloff (F) \\
\hline S_28 & $\begin{array}{c}25 \% \text { MARAVA } \\
30 \% \text { BOKSER } \\
30 \% \text { WADI }\end{array}$ & - & $15 \%$ Phleum pratense & Equitana Nachsaat Gvo, Rudloff (F) \\
\hline S_29* & $10 \%$ TURFGOLD & $\begin{array}{l}45 \% \text { BARCESAR, } \\
25 \% \text { DEBUSSY } 1\end{array}$ & $20 \%$ Poa pratensis & Monaco-Mischung RSM, Eurogreen $(\mathrm{T})$ \\
\hline S_30 & $\begin{array}{c}11 \% \text { TREND } \\
5 \% \text { KARATOS } \\
10 \% \text { TWYMAX }\end{array}$ & - & $\begin{array}{c}10 \% \text { Festuca pratensis, } \\
11 \% \text { Festulolium fedoro, } \\
7 \% \text { Dactylis glomerata, } \\
5 \% \text { Poa pratensis, } \\
5 \% \text { Festuca rubra, } \\
14 \% \text { Phleum pratense }\end{array}$ & Kräuterweide, Camena Samen (F) \\
\hline S_31 & $\begin{array}{l}\text { Unknown variety in unknown } \\
\text { percentage }\end{array}$ & - & $\begin{array}{c}\text { Festuca pratensis, Poa pratensis, Poa } \\
\text { trivialis, Festuca rubra, Phleum } \\
\text { pratense, Alopecurus pratensis, } \\
\text { Cynosurus cristatus, Elymus repens }\end{array}$ & $\begin{array}{c}\text { Pferdeweide-Reparatursaat, } \\
\text { Kräuterwiese }(\mathrm{F})\end{array}$ \\
\hline S_32 & $\begin{array}{c}15 \% \text { MARAVA } \\
15 \% \text { BOKSER } \\
15 \% \text { WADI }\end{array}$ & - & $\begin{array}{c}25 \% \text { Phleum pratense } \\
20 \% \text { Poa pratensis } \\
10 \% \text { Festuca rubra }\end{array}$ & Equitana Universal, Rudloff (F) \\
\hline S_33* & $\begin{array}{c}\mathbf{9} \% \text { COLUMBINE } \\
7 \% \text { DOUBLE/FABIAN } \\
12 \% \text { ZURICH } \\
5 \% \text { CSI CORSICA } \\
\mathbf{1 2} \% \text { SIRTAKY }\end{array}$ & - & $\begin{array}{l}40 \% \text { Poa pratensis, } \\
15 \% \text { Festuca rubra }\end{array}$ & Primera Highspeed, UFA (T) \\
\hline
\end{tabular}

* Seed composition as indicated on package different to that in online catalogs. \# Seed composition not indicated on package. 
These inconsistencies occurred as the information on seed varieties were wrongly taken from catalogs instead of package labels. We want to point out that contaminations with Epichloë infected seeds could have occurred at different production steps of the seed mixtures (breeders, producer of seed cultivars, producer of the seed mixture, trader, shop/market, etc.). Therefore, our study cannot be used to detect the source of the Epichloë contaminations of the seed varieties.

The authors want to point out that seed mixture S_33 did not contain the perennial ryegrass variety NEW ORLEANS, which was therefore not tested. Speculations on possible infections of the variety NEW ORLEANS in the discussion are not valid and we apologize for this unjustified speculation.

Therefore the authors would like to delete these sentences from the discussion: "The turfgrass seed mixture S_33 contains 5\% of the perennial ryegrass variety NEW ORLEANS, which is listed as a top American breeding variety and could be the source of Epichloë infected seeds. The low percentage of this variety in the seed mixture could result in sampling variation, which may explain why $8.7 \%$ of E+ seeds were detected, and why the alkaloid quantification results differed between the two laboratories."

Following a reviewer's comment, we meanwhile grew plants from the four grass seed mixtures (S_10, S_24, S_32 and S_33) which were Epichloë infected and contained alkaloids, and tested 8- and 20-week old seeded plants for Epichloë alkaloids. We used 30 randomly picked seeds per seed mixture and tested eight grass samples containing about ten randomly picked grass tillers per seed mixture. In S_10 and S_33 we detected peramine and lolitrem B. In S_32, we detected peramine, lolitrem B and in addition ergovaline. In S_24, we could not detect alkaloids in the plants. As we only picked 30 random seeds, it could be possible that no infected seeds were grown from S_24. As three of the four endophyte and alkaloid positive seed mixtures produced alkaloids in the grown plants, we confirm our result that infected seed mixtures can introduce vertebrate toxic alkaloids into the agricultural environment.

The authors would like to apologize for any inconvenience caused to the readers by these changes.

Funding: This research received no external funding.

Conflicts of Interest: The authors declare no conflict of interest.

\section{References}

1. Krauss, J.; Vikuk, V.; Young, C.A.; Krischke, M.; Mueller, M.J.; Baerenfaller, K. Epichloë endophyte infection rates and alkaloid content in commercially available grass seed mixtures in Europe. Microorganisms 2020, 8, 498. [CrossRef] [PubMed]

Publisher's Note: MDPI stays neutral with regard to jurisdictional claims in published maps and institutional affiliations.

(C) 2020 by the authors. Licensee MDPI, Basel, Switzerland. This article is an open access article distributed under the terms and conditions of the Creative Commons Attribution (CC BY) license (http://creativecommons.org/licenses/by/4.0/). 